\title{
Environmental Influence on Microwave Radiometry for Buried Object Detection
}

\author{
David R. Wiggins, Baran U. Ungan, and Joel T. Johnson \\ ElectroScience Laboratory, The Ohio State University \\ 1320 Kinnear Rd., Columbus, OH 43212 \\ Phone: (614) 292-7981, Email: wiggins.38@osu.edu
}

\section{Introduction}

Microwave radiometers have been shown to be effective sensors for monitoring soil moisture and other geophysical data [1]-[4]. Models for geophysical medium brightness temperatures often consider horizontally stratified geometries, because exact evaluation of observed brightness temperatures is possible for this case in terms of a sum involving layered medium reflection quantities [2]. For cases involving media whose temperature is constant, the formulation reduces to "Kirchhoff's law" which relates emissivity to one minus the reflection coefficient of the layered medium. Previous studies of microwave radiometry for soil moisture remote sensing have considered both temperature and soil moisture variations with depth, and shown that these environmental effects can have important influences on soil moisture retrievals [3].

Recent studies have begun to consider the use of microwave radiometers for detecting shallow, sub-surface objects such as anti-personnel landmines [5]-[6]. Modeling studies in these references based on Kirchhoff's law and a three layer, horizontally stratified geometry (i.e. objects are infinite layers) show that significant brightness temperature contrasts can be obtained in the presence of a sub-surface object if sufficient dielectric contrast is available and if soil medium attenuation is not excessive. Reference [6] further demonstrated that use of multi-frequency brightness measurements could potentially provide detections even with low contrast or high attenuation due to the oscillatory behavior versus frequency observed in the presence of an object. Estimated environmental effects such as local surface temperature or soil moisture variations would not produce oscillatory frequency behavior (except in unusual circumstances [4]) so that detections would still be possible even in the presence of environmental "clutter".

However, previous buried object detection models have neglected variations in medium temperature (due to the use of Kirchhoff's law) and soil moisture (which was assumed constant) with depth. Since these factors can significantly impact soil mositure remote sensing, their effect on buried object detection requires consideration as well. In this paper, a multi-layer horizontally stratified emission model is coupled with a numerical solution of the heat equation and a model for water transport in the presence of a sub-surface object to estimate the importance of temperature and soil moisture variations. Results show that these factors can impact overall brightness temperatures, but that the concept of using oscillatory features in frequency swept data as an indicator of a sub-surface object remains valid.

\section{Moisture, Thermal, and Emission Models}

Figure 1 illustrates the geometry considered: a $6.8 \mathrm{~cm}$ thick subsurface object (modeled as a layer of constant relative permittivity $3+i 0.08$ ) is located $4.25 \mathrm{~cm}$ below an airsoil boundary. Both permittivity $\epsilon(z)$ and temperature $T(z)$ are not constant in the soil background medium, and the temperature also varies in the object layer. Specification of the medium begins with a moisture transport model [7] for evaluating soil volumetric moisture contents in the presence or absence of a sub-surface object of dimensions $25.2 \times 25.2 \times 6.8 \mathrm{~cm}$. Water transport is computed on a grid $73.5 \times 73.5 \times 59.5 \mathrm{~cm}$ in the presence of a periodic "rain" forcing function. Further details of the simulation are provided in [8]. Figure 2, plot (a) illustrates the resulting moisture profiles versus depth with (taken from directly 


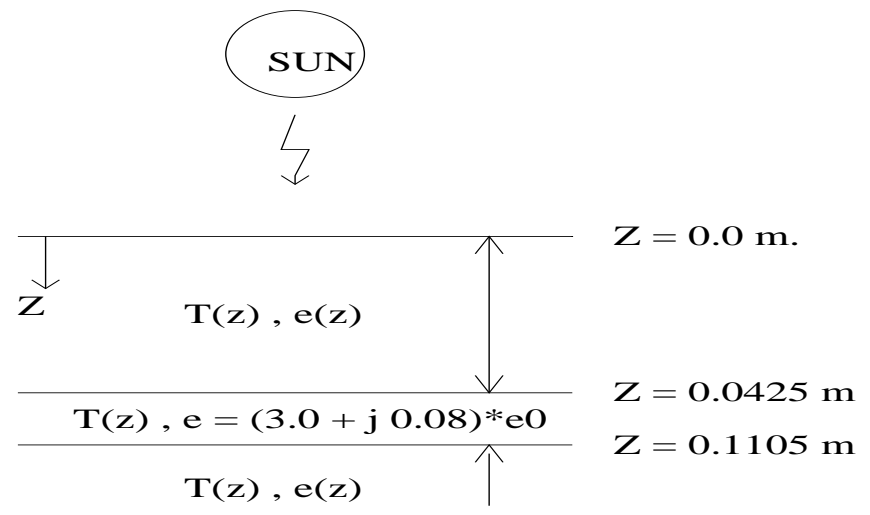

Figure 1: The stratified medium model

underneath the object) and without (taken from one of the faces of the computational domain) the object; note the increased and decreased water contents above and below the object respectively. Moisture contents versus depth then directly determine the permittivity profile $\epsilon(z)$ through the empirical soil permittivity model of [1] evaluated for sand with bulk density $1.12 \mathrm{~g} / \mathrm{cm}^{3}$. Obtained permittivity values ranged from $5.44+i 0.46$ to $12.74+i 2.15$; note the significant contrast with the subsurface object, although high attenuation is also obtained for this case.

Moisture contents are also used to determine the thermal conductivity and diffusivity parameters of the soil medium versus depth from the model of [9]. A one dimensional solution of the heat equation with or without the object layer is then used to determine the temperature profile versus depth as described in [10]. Parameters for solar forcing, wind convection, and other effects are as described in [10]. The heat equation is solved on a 100 layer grid up to depth $50 \mathrm{~cm}$, beyond which the temperature is assumed constant. Figure 2, plot (b) illustrates typical temperature profiles obtained with and without the subsurface object at local time $15: 42$. In these simulations the subsurface object is modeled thermally as a near-insulator, allowing the relatively large temperature difference through the object layer to be maintained.

Finally, given the $\epsilon(z)$ and $T(z)$ profiles versus depth, brightness temperatures are computed from the multi-layer fluctuation-dissipation model of [2]. This model includes all potential coherent emission effects, and again is evaulated on the 100 layer grid up to depth $50 \mathrm{~cm}$. Results to be illustrated consider nadir observation, so that polarization effects can be neglected. Results are compared with computations which neglect either temperature variations (by assuming the entire medium is at the surface temperature) or permittivity variations (by assuming the soil medium is all of uniform permittivity) to clarify the influence of these factors.

\section{Results}

Figure 3 compares brightness temperatures from the complete model with the object versus those assuming $T(z)=T_{s}=333 \mathrm{~K}$ or $\epsilon(z)=\epsilon$ (computed for $27 \%$ soil moisture). Results are plotted as a function of radiometer frequency from 1 to $5 \mathrm{GHz}$, and the oscillatory pattern versus frequency obtained in the presence of a subsurface object is observed. The complete model with no object is also included, and shows no strong oscillations versus frequency. For this relatively high attenuation case, the influence of temperature variations is observed to be small, since greater depths at which temperature changes are larger are not observed by the radiometer. Permittivity variations are found to have a larger effect, but the basic concept of searching for oscillatory features for object detection remains valid.

To illustrate an additional case with lower attenuation, the moisture profiles of Figure 2 are divided by 4 to obtain an average moisture content of $6.7 \%$, and the thermal and 
(a)

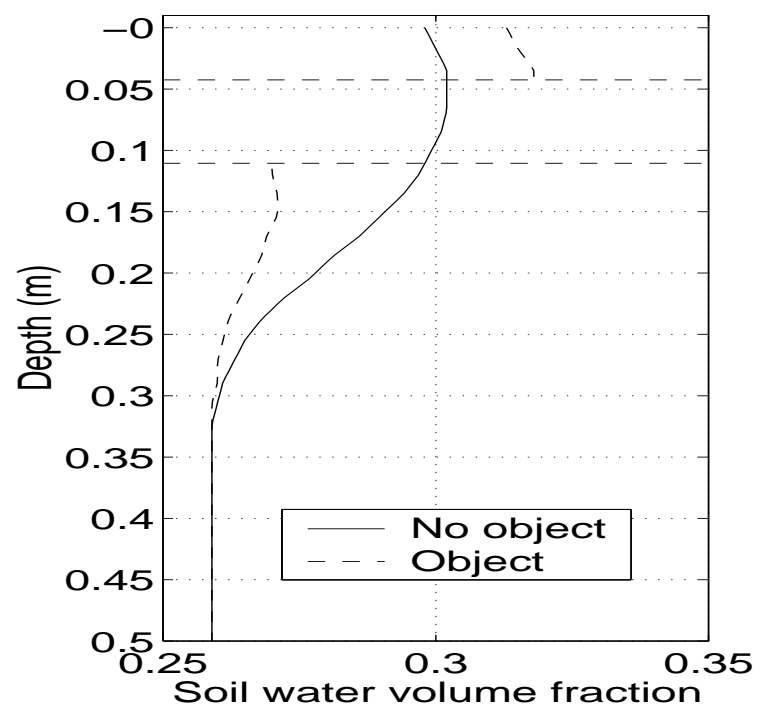

(b)

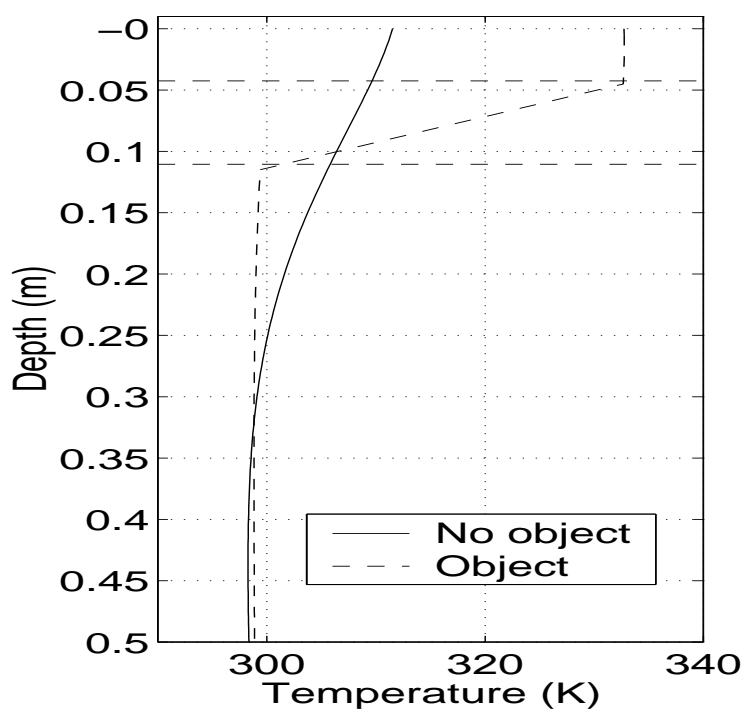

Figure 2: Moisture (a) and temperature (b) profiles with and without object

emission models re-computed. Results are illustrated in Figure 4, and show smaller oscillations due to the reduced dielectric contrast but a greater influence of both temperature and permittivity profile variations. However, the basic features with and without the subsurface object again remain similar.

The results of this study confirm that temperature and moisture profiles can influence brightness temperatures, but that these factors are unlikely to produce the oscillatory features caused by a subsurface object.

\section{References}

[1] F. T. Ulaby, R. K. Moore and A. K. Fung, Microwave Remote Sensing: Active and Passive, Norwood, MA: Artech House, 1986.

[2] L. Tsang, J. A. Kong, and R. T. Shin, Theory of Microwave Remote Sensing, New York: John Wiley and Sons, 1985.

[3] Y.-A. Liou and A. W. England, "A land surface process/ radiobrightness model with coupled heat and moisture transport in soil," IEEE Trans. Geosc. Rem. Sens., vol. 36, pp. 273-286, 1998.

[4] T. J. Schmugge, T. J. Jackson, P. E. O'Neill, and M. B. Parlange, "Observations of coherent emissions from soils," Radio Sci., vol. 33, pp. 267-272, 1998.

[5] G. De Amici, B. Hauss, and L. Yujiri, "Detection of landmines via a passive microwave radiometer", SPIE Aerosense meeting: Detection and Remediation Technologies for Mines and Mine-Like Targets IV, conference proceedings, 1999.

[6] J. T. Johnson, "Theoretical study of microwave radiometry for buried object detection," SPIE Aerosense meeting: Detection and Remediation Technologies for Mines and Mine-Like Targets $V$, conference proceedings, 2000.

[7] J. Simunek, K. Huang, and M. van Genuchten, "The SWMS-3D code for simulating water flow and solute transport in three dimensional variably saturated media," Research Report No. 139, U. S. Salinity Laboratory, USDA, 1995. 




Figure 3: Brightness temperatures $\left(T_{B}\right)$ vs. frequency for the high moisture case

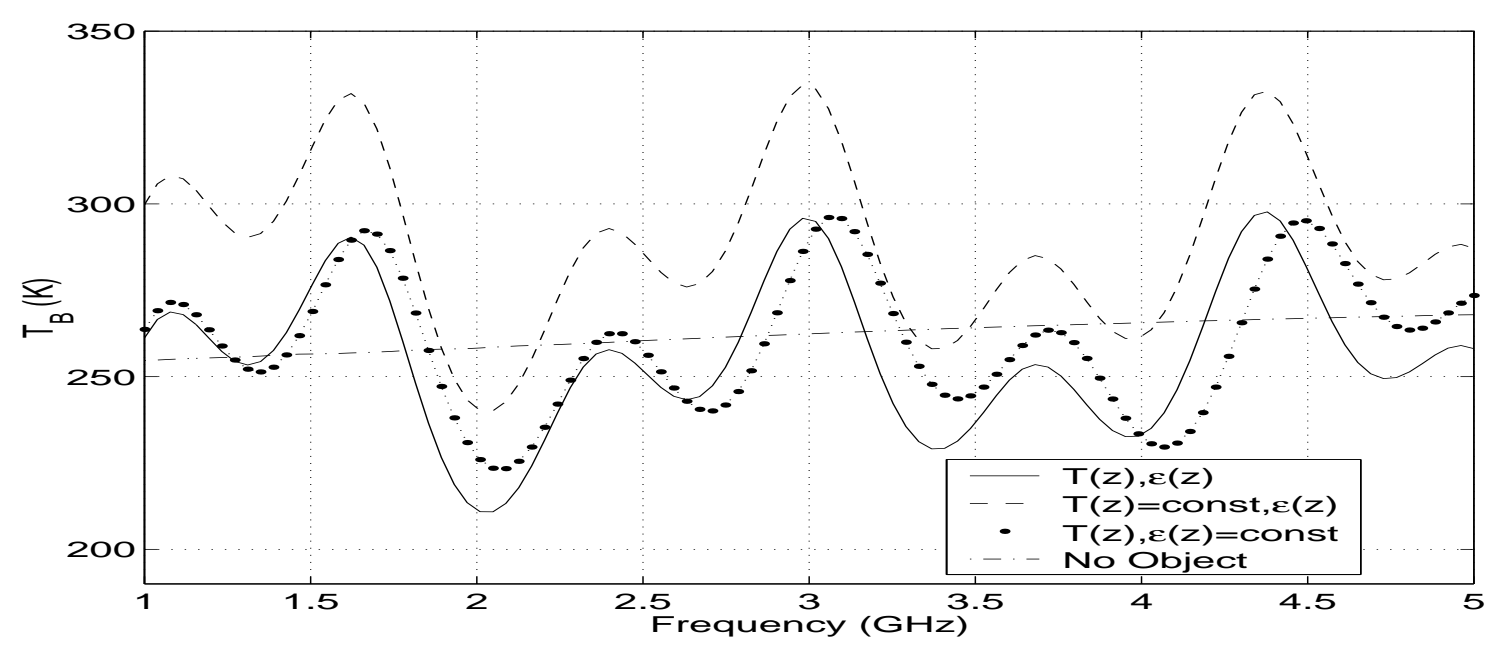

Figure 4: Brightness temperatures $\left(T_{B}\right)$ vs. frequency for the reduced moisture case

[8] B. A. Baertlein and I. K. Sendur, "The role of environmental factors and mine composition in thermal IR mine signatures," submitted to SPIE Aerosense meeting: Detection and Remediation Technologies for Mines and Mine-Like Targets VI, 2001.

[9] P. Pregowski, W. Swiderski, R. T. Walczak, and K. Lamorski, "Buried mine and soil temperature prediction by numerical model,' SPIE Aerosense meeting: Detection and Remediation Technologies for Mines and Mine-Like Targets V, conference proceedings, 2000 .

[10] I. K. Sendur and B. A Baertlein. "Techniques for improving buried mine detection in thermal IR imagery," SPIE Aerosense meeting: Detection and Remediation Technologies for Mines and Mine-Like Targets IV, conference proceedings, 1999. 